\title{
ON THE UNIFORM CONTINUITY OF WIENER PROCESS WITH A MULTIDIMENSIONAL PARAMETER
}

\author{
TAKEYUKI HIDA
}

\section{§1. Introduction}

Let $X(A, \omega), \omega \in \Omega$, be Wiener process on the probability space $(\Omega, \mathfrak{B}, P)$ depending on a point $A$ of an $N$ dimensional Euclidean space $\mathbf{E}_{\mathbf{N}}$. It is defined by the following three conditions:

(1) For any positive integer $n$ and any points $A_{1}, A_{2}, \ldots, A_{n}$ in $\mathbf{E}_{\mathbf{N}}$, the joint distribution of $\left\{X\left(A_{1}\right), X\left(A_{2}\right), \ldots, X\left(A_{n}\right)\right\}$ is a non-degenerate $n$ dimensional normal distribution with mean vector $\mathbf{O}$.

$$
E\{X(A) X(B)\}=\{r(O, A)+r(O, B)-r(A, B)\} / 2,
$$

where $r(A, B)$ denotes the distance between $A$ and $B$, and $O$ denotes the origin of $\mathbf{E}_{\mathbf{N}}$.

$$
X(O, \omega)=0, \quad \text { for almost every } \omega \in \Omega .
$$

Previously P. Lévy studied this process in his book [1] and obtained many properties of it. Concerning the uniform continuty, he proved the following:

If $a>1$, for almost every $\omega \in \Omega$, there exists a positive number $\rho=\rho(\omega)$ such that

$$
r=r(A, B)<\rho \quad \text { implies }|X(A, \omega)-X(B, \omega)| \leqq a \sqrt{ } 2 N r|\log r| .
$$

On the other hand, if $a<1$, there exists no such $\rho(\omega)$. Here both $A$ and $B$ are arbitrarily chosen in the cube $C=\left\{A=\left(a_{1}, a_{2}, \ldots, a_{N}\right) ;\left|a_{i}\right| \leqq 1, i=1,2, \ldots, N\right\}$.

While $\mathrm{T}$. Sirao [2] has proved a very precise continuity property of Wiener process with a parameter space $\mathbf{E}_{\mathbf{1}}$. It is stated as follows:

If $c>5$, there exists a positive number $\rho=\rho(\omega)$ such that

$$
r=\left|t^{\prime}-t\right|<\rho \text { implies }\left|X(t, \omega)-X\left(t^{\prime}, \omega\right)\right| \leqq \sqrt{ } 2 r(|\log r|+c \log |\log r|),
$$

with probability one. Here $t$ and $t^{\prime}$ are arbitrary numbers in the interval $[0,1]$. But, for $c<-1$, there exists no such $\rho(\omega)$.

Received December 10, 1957. 
The aim of the present note is to improve the Lévy's result employing the method used by Sirao [2]. Our result may be regarded as the extension of the Sirao's result for the multidimensional parameter space. But, in our case where the paramter space is $\mathbf{E}_{\mathbf{N}}$, we shall consider the $n$-th bundle of lines and every variation of $X(A)$ relating to it as will be seen at the proof of Theorem 1. Moreover, for the proof of Theorem 2 that gives the lower estimation of our process, we shall use the lemma and the method of the proof of Theorem 1 which appear in the paper written by K. L. Chung and P. Erdös [3].

\section{§ 2. Upper estimation}

Before we state the theorem, some preparations are necessary. Generally the direction of any straight line that runs through the origin is determined by the ordered set of angles $\left(\theta_{1}, \theta_{2}, \ldots, \theta_{x-1}\right), 0 \leqq \theta_{i}<\pi, i=1,2, \ldots, N-1$. We consider $n^{N-1}$ straight lines the direction of which are given such as $\left(j_{1} \pi / n^{2}, j_{2} \pi / n^{2}, \ldots, j_{N-1} \pi / n^{2}\right), j_{k}=0,1, \ldots, n^{2}-1, k=1,2, \ldots, N-1$.

Taking one of those straight lines $\varnothing$, we consider the hyper plane $\mathscr{S}$ that is orthogonal to $\&$ and contains the origin. On this hyper plane we take $\left(2^{n}+1\right)^{v-1}$ lattice points, the coordinates of which are such as $\left(j_{1} / 2^{n-1}, j_{2} / 2^{n-1}\right.$, $\left.\ldots, j_{Y-1} / 2^{n-1}\right), j_{k}=0, \pm 1, \pm 2, \ldots, \pm 2^{n-1}, k=1,2, \ldots, N-1$, for suitably chosen coordinate system. And then, for every one of those lattice points, we consider a straight line that is parallel with \&s and runs through that point. The figure that consists of the hyper plane $\mathfrak{H}$ and $\left(2^{n}+1\right)^{N-1}$ straight lines considered above will be called the $n$-th bundle of lines and will be denoted by $\mathfrak{Q}_{n}$.

In a similar way, we have $n^{2 N-2} n$-th bundles of lines. The lattice point of $\Omega_{n}$ is defined as the point that lies on some straight line of $\Omega_{n}$ at the distance of $k / 2^{n-1}$ ( $k$ is an integer and $|k| \leqq 2^{n-1}$ ) from the corresponding hyper plane. Thus $\mathfrak{\Omega}_{n}$ has $\left(2^{n}+1\right)^{x}$ lattice points.

For any pair of lattice points $A_{1}$ and $A_{2}$ of $\Omega_{n}$ such that they lie on the same straight line and $r\left(A_{1}, A_{2}\right)=1 / 2^{n-1}$, there corresponds the difference $\Delta X(A)=X\left(A_{1}\right)-X\left(A_{2}\right)$, which we shall call the variation of $X(A)$ relating to $\mathfrak{Z}_{n}$.

Let us put 


$$
\varphi_{c}(r)=\{r(2 N|\log r|+c \log |\log r|)\}^{1 / 2} .
$$

It is easily seen that $\varphi_{c}(r)$ is monotone for small $r>0$ and tends to 0 as $r \rightarrow+0$.

Thus we may state the following theorem for Wiener process $X(A)$, when $A$ runs in the unit sphere of $\mathbf{E}_{\mathbf{N}}$ with center $O$.

THEOREM 1. If $c>8 N+1$, for alnost every $\omega$, there exists a positive number $\rho=\rho(\omega)$ such that

$$
r=r(A, B)<\rho \quad \text { implies }|X(A)-X(B)| \leqq \varphi_{c}(r),
$$

Proof. Let us put $c=8 N+1+\varepsilon$ with a positive number $\varepsilon$. First we take one of the straight lines belonging to $\mathfrak{Z}_{n}$. The lattice points lying on it are arranged as $A_{0}, A_{1}, \ldots, A_{2^{n}}$ in a order of their position. It is noted that on that straight line $X(A)$ may be considered as a usual Wiener process with a linear parameter.

We shall define $\alpha_{n, n, l}$ as the probability that the inequality

$$
\begin{aligned}
\left|X\left(A_{m+l}\right)-X\left(A_{l}\right)\right|>\varphi_{c}\left(m / 2^{n-1}\right), & m=1,2, \ldots,\left[n^{2} \log n\right], \\
l & =0,1, \ldots, 2^{n}-1,
\end{aligned}
$$

holds. Since $\alpha_{n, m, l}$ is independent of $l$, we may write simply $\alpha_{n, m}$. Then we have

$$
\alpha_{n, 1} \leqq \alpha_{n, 2} \leqq \ldots \leqq \alpha_{n,\left[n^{2} \log n\right]}
$$

for sufficiently large $n$ (See Sirao [2]).

Noting that $X(A)-X(B)$ is a normal random variable with mean 0 and variance $r=r(A, B)$, we have the following asymptotic relation.

$$
P\left(|X(A)-X(B)|>\varphi_{c}(r)\right)=O(1) r^{N}(|\log r|)^{-(c+1) / 2},
$$

for sufficiently small $r>0$. Therefore we have

$$
\alpha_{n,\left[n^{2} \operatorname{lng} n\right]}=O(1) 2^{-n x} n^{-(2 x+1+\varepsilon / 2)}(\log n)^{N} .
$$

If we consider such a probability for every straight line belonging to $\Omega_{n}$, in a similar way, we have

$$
\begin{aligned}
& \sum_{n} n^{2 N-2}\left(2^{n}+1\right)^{x-1} n^{2}(\log n) 2^{n}\left\{O(1) 2^{-n x} n^{-(2 N+1+\varepsilon / 2)}(\log n)^{x}\right\} \\
& \quad=O(1) n^{-(1+\varepsilon / 2)}(\log n)^{N+1}<\infty .
\end{aligned}
$$

The $n$-th term of the left hand side of (10) is not less than the probability 
that there exists at least one straight line belonging to $\Omega_{n}$ and exists at least one pair $(m, l)$ satisfying the inequality $(7)$. The convergence property of (10) shows, by Borel-Cantelli's Lemma, that there exists a positive integer $n_{0}=n_{0}(\omega)$ such that $n>n_{0}(\omega)$ implies the validity of (6) for every variation relating to $\Omega_{n}$ with probability one. Hence the theorem is proved for the particular case where both $A$ and $B$ are the lattice points lying on the same straight line of $\mathfrak{L}_{n}\left(n>n_{0}(\omega)\right)$ and $r(A, B)=m / 2^{n-1}, m=1,2, \ldots,\left[n^{2} \log n\right]$.

Next we shall prove the theorem in a general case. For any points $A$ and $B$ in the unit sphere with center $O$, if $r=r(A, B)$ is sufficiently small, there exists a positive integer $n$ such that

$$
\left[n^{2} \log n\right] / 2^{n}<r \leqq\left[(n-1)^{2} \log (n-1)\right] / 2^{n-1} .
$$

Here $r$ must be very small so that $n>n_{0}(\omega)$. Then there is some straight line $\$$ belonging to one of the $n$-th bundles of lines that is the nearest one to the segment $A B$. Let $A_{1}$ and $B_{1}$ be the projections of $A$ and $B$ on $\varnothing$ respectively. Then there are some lattice points $A_{2}$ and $B_{2}$ lying on $\varnothing$, which are the nearest ones to $A_{1}$ and $B_{1}$ respectively and satisfy the inequality $r\left(A_{2}, B_{2}\right) \leqq r(A, B)$. These facts imply

$$
\begin{gathered}
r\left(A_{2}, B_{2}\right)=m / 2^{n-1}, \\
r_{A}=r\left(A, A_{2}\right)<c_{1} \log n / 2^{n-1} \text { and } r_{B}=r\left(B, B_{2}\right)<c_{2} \log n / 2^{n-1},
\end{gathered}
$$

where $c_{1}$ and $c_{2}$ are absolute constants and $m$ is an integer that is not less than $\left[2^{-1} n^{2} \log (n-1)\right]$.

Let us put $c^{\prime}=c-\varepsilon / 2$. Then $c^{\prime}>8 N+1$. Since $n>n_{0}(\omega)$, by using the above conclusion for the particular case, we have

$$
\left|X\left(A_{2}\right)-X\left(B_{2}\right)\right| \leqq \varphi_{c^{\prime}}\left(m / 2^{n-1}\right) \leqq \varphi_{c}(r) .
$$

The second inequality is derived from the monotony of $\varphi_{c}(r)$. Therefore we can prove, from (4) and (13),

$$
\begin{aligned}
|X(A)-X(B)| & \leqq\left|X\left(A_{2}\right)-X\left(B_{2}\right)\right|+\left|X(A)-X\left(A_{2}\right)\right|+\left|X(B)-X\left(B_{2}\right)\right| \\
& \leqq \varphi_{c^{\prime}}(r)+a \sqrt{2} N\left(\sqrt{r_{A}}\left|\log r_{A}\right|+\sqrt{\left.r_{B}\left|\log r_{B}\right|\right)}\right. \\
& <\sqrt{ } r\left(2 N|\log r|+c^{\prime} \log |\log r|\right)+b \sqrt{n \log n / 2^{n}}
\end{aligned}
$$

where $b$ is a constant determined by $c_{1}, c_{2}$ and $a$. Noting that $r$ is of order $\left(n^{2} \cdot \log n\right) / 2^{n}$, we can easily prove by the simple computations that the last side of (14) is less than $\varphi_{c}(\boldsymbol{r})$. Thus we have completely proved the theorem. 


\section{§. Lower estimation}

Fist let us state the theorem.

THEOREM 2. If $c<1$, for almost every $\omega$, there exist infinitely many pairs $(A, B)$ in $C$ such that the inequality

$$
|X(A)-X(B)|>\varphi_{c}(r), \quad(r=r(A, B)),
$$

holds.

Before proceeding to the proof of this theorem, we shall state some simple lemmas.

Lemma 1. Let $\left\{F_{k}\right\}, k=1,2, \ldots, n$, be an arbitrary sequence of events in $(\Omega, \mathfrak{B}, P)$. Then we have, if $P\left(\bigcup_{k=1}^{u} F_{k}\right)>0$,

$$
2 \sum_{1 \leqq j<k \leqq n} P\left(F_{j} \cap F_{k}\right) \gtrsim\left[P\left(\bigcup_{k=1}^{n} F_{k}\right)\right]^{-1}\left(\sum_{k=1}^{n} P\left(F_{k}\right)\right)^{2}-\sum_{k=1}^{n} P\left(F_{k}\right) .
$$

The proof is found in K. L. Chung and P. Erdös [3].

Lemma 2. Let $\Delta_{1} X(A)=X\left(A_{1}\right)-X\left(A_{1}^{\prime}\right)$ and $\Delta_{2} X(A)=X\left(A_{2}\right)-X\left(A_{2}^{\prime}\right)$ be arbitrary variations relating to the $n$-th and $(n+m)$-th bundles of lines respectively and assume that $A_{1} A_{1}^{\prime}$ and $A_{2} A_{2}^{\prime}$ be parallel with each other. Then, if the distance between two segments is longer than $n / 2^{n-1}$, the correlation coefficient $r_{1,2}$ of $\Delta_{1} X(A)$ and $\Delta_{2} X(A)$ is less than $\left(\lambda_{n} \lambda_{n+m}\right)^{-1}$. Here $\lambda_{k}$ is defined by

$$
\lambda_{k}=2^{(k-1) / 2} \varphi_{c}\left(1 / 2^{k-1}\right) .
$$

Considering the results obtained by P. Lévy (See $[1] \S 61$ ), we can prove this lemma by elementary considerations.

Lemma 3. Let $\Delta_{1} X(A)$ and $\Delta_{2} X(A)$ be what were defined in Lemma 2 and $n$ be large. Let us put

(18) $E_{1}=\left\{\omega ; \Delta_{1} X(A)>\varphi_{c}\left(1 / 2^{n-1}\right)\right\}$ and $E_{2}=\left\{\omega ; A_{2} X(A)>\varphi_{c}\left(1 / 2^{n+m-1}\right)\right\}$.

Then, if $r_{1,2}<\left(\lambda_{n} \lambda_{n+m}\right)^{-1}$, we can find an absolute constant $d$ satisfying

$$
P\left(E_{1} \cap E_{2}\right)<d P\left(E_{1}\right) P\left(E_{2}\right) .
$$

And, if $\left(\lambda_{n} \lambda_{n+m}\right)^{-1} \leqq r_{1,2} \leqq 1 / 2$, we have

$$
P\left(E_{1} \cap E_{2}\right) \leqq O(1) e^{-\alpha ! n+m)} P\left(E_{1}\right)
$$

for some $\alpha>0$. 
Proof. By the asymptotic relation (8), it is proved that

$$
P\left(E_{1}\right) P\left(E_{2}\right)=O(1)\left(\lambda_{n} \lambda_{n+m}\right)^{-1} e^{\left.-\left(\lambda_{n t}^{2}+\lambda_{n+m}\right)^{2}\right) / 2} .
$$

Under the assumption $0<r_{1,2}<\left(\lambda_{n} \lambda_{n+m}\right)^{-1}$, the left side of (19) becomes

$$
\begin{aligned}
& P\left(E_{1} \cap E_{2}\right)=(2 \pi)^{-1}\left(1-r^{2}\right)^{-1 / 2} \int_{\lambda_{n}}^{\infty} \int_{\lambda_{n+m}}^{\infty} e^{-\left(x^{2}+y^{2}-2 r x y\right) / 2\left(1-r^{2}\right)} d x d y \quad\left(r=r_{1,2}\right) \\
& =(2 \pi)^{-1}\left(1-r^{2}\right)^{-1 / 2} \int_{\lambda_{n}}^{\gamma_{3} \lambda_{n+m}} \int_{\lambda_{n+m}}^{\gamma_{3} \lambda_{n+m}} e^{-(x-r y)^{2} / 2} e^{-y^{2} / 2} d x d y \\
& +O\left(\frac{1}{\lambda_{n+m}} e^{-\lambda_{n+m^{2}}}\right) \\
& \leqq O(1) \lambda_{n+m}^{-1} e^{-\lambda_{n+m^{2} / 2}} \int_{\lambda_{n}}^{\gamma_{3} \lambda_{n+m}} e^{-\left(x-\gamma^{\overline{2}} / \lambda_{n}\right)^{2} / 2} d x \\
& =O(1)\left(\lambda_{n} \lambda_{n+m}\right)^{-1} e^{-\left(\lambda^{2}{ }_{n}+\lambda^{2}{ }_{n+m}\right) / 2}
\end{aligned}
$$

If $r_{1,2} \leqq 0,(19)$ is a trivial inequality. Thus, from (21) and (22), we can find $d$ satisfying the inequality (19).

If $r_{1,2} \leqq 1 / 2$, from the second equality of $(22)$, we have

$$
\begin{aligned}
P\left(E_{1} \cap E_{2}\right) & \leqq O(1) \lambda_{n}^{-1} e^{-\lambda^{2} n^{2}} \lambda_{n+m}^{-1} e^{-\left(\lambda^{2} n+m^{\left.\left(1-V_{3 / 2}\right)^{2}\right) / 2\left(1-r^{2}\right)}\right.} & \\
& \leqq O(1) e^{-\alpha(n+m)} P\left(E_{1}\right), & (\alpha>0),
\end{aligned}
$$

which proves the inequality (20).

Proof of Theorem 2. ${ }^{1)}$ For every variation $\Delta_{n, j} X(A), j=1,2, \ldots, 2^{n N}$, relating to the $n$-th bundle of lines, ${ }^{2)}$ let us put

$$
E_{n, j}=\left\{\omega ; \Delta_{n, j} X(A)>\varphi_{c}\left(1 / 2^{n-1}\right)\right\}, \quad j=1,2, \ldots, 2^{n}\left(2^{n}+1\right)^{N-1} .
$$

Then we have

$$
\sum_{n} \sum_{j} P\left(E_{n, j}\right)=O(1) \sum_{n} 2^{n N}\left(2^{-n N} n^{-(1+c) / 2}\right)=\infty,
$$

from the assumption $c<1$. Let us put

$$
E_{n}=\bigcup_{j} E_{n, j} \text { and } B_{m}=\bigcup_{n=m}^{\infty} E_{n}
$$

In order to show that $\lim _{\infty \rightarrow m} P\left(B_{m}\right)=1$, we shall prove that $P\left(B_{m}\right)=1$ for everv $m$.

Suppose $P\left(B_{m}\right)<1$ for some $m$. And let $P\left(B_{m}\right)=1-\delta,(\delta>0)$. Then we have

1) We owe the method of this proof to K. L. Chung and P. Erdös. But we need some modifications.

2) Hereafter, for every $n$, we take one of the $n$-th bundles of lines that contains the same hyper plane. 


$$
P\left(B_{m}^{c}\right)=P\left(\bigcap_{n=m}^{c \infty} E_{n}^{c}\right)=\delta .
$$

For any $\varepsilon$ such that $1-\delta>\varepsilon>0$, there exists an integer $l$ satisfying the following inequality:

$$
P\left(\bigcup_{n=m}^{l} E\right)>1-\delta-\varepsilon .
$$

If we write $G_{m, l}=B_{m}-\bigcup_{n=m}^{l} E_{n}$, we have

$$
P\left(G_{m, l}\right)<\varepsilon
$$

As is easily seen, for the given event $\bigcup_{n=m}^{l} E_{n}$, there exists a large $K(>m)$ such that

$$
P\left(E_{k, p} \mid\left(\bigcup_{n=m}^{l} E_{n}\right)^{c}\right)>{ }_{2}^{1} P\left(E_{k, p}\right)
$$

holds for any $k>K$ and any $p>0$. Hence, from (24) and (28), we have

$$
\sum_{k} \sum_{p} P\left(E_{k, p} \cap\left(\bigcup_{n=m}^{l} E_{n}\right)^{c}\right)=\infty \text {. }
$$

Therefore we can choose an integer $K^{\prime}>K$ such that

$$
1<\sum_{k=K}^{K^{\prime}} \sum_{p} P\left(E_{k, p} \cap\left(\bigcup_{n=m}^{l} E_{n}\right)^{c}\right) \leqq 2,
$$

where the sum $\sum_{v}$ should be properly added when $k=K$ or $=K^{\prime}$. Thus we obtain, from (25), (28) and (29),

$$
\sum_{k=K^{\prime}}^{K^{\prime}} \sum_{p} P\left(E_{k, p}\right)<4 / \delta
$$

Furthermore we consider the following relation:

$$
\text { (31) } \begin{aligned}
\sum_{K \leqq k<k^{\prime} \leqq K^{\prime}} \sum_{p, p^{\prime}} P\left(E_{k, p} \cap E_{k^{\prime}, p^{\prime}}\right) \leqq & \sum^{1} P\left(E_{k, p} \cap E_{k^{\prime}, p^{\prime}}\right)+d \sum^{2} P\left(E_{k, p}\right) P\left(E_{k^{\prime}, p^{\prime}}\right) \\
& +\sum_{k=K}^{K^{\prime}} P\left(E_{k, p} \cap E_{k+1, p^{\prime}}\right),
\end{aligned}
$$

where $\Sigma^{1}$ or $\sum^{2}$ indicates the summation extending over those $k, k^{\prime}, p$ and $p^{\prime}$ for which the corresponding correlation coefficient $r$ satisfies the inequality $r<\left(\lambda_{k} \lambda_{k^{\prime}}\right)^{-1}$ or $1 / 2 \gtrsim r \gtrsim\left(\lambda_{k} \lambda_{k}\right)^{-1}$ respectively, and $\sum^{3}$ indicates the summation of the rest, that is, the case $r>1 / 2$.

The sum $\sum^{3}$ is not larger than $2 \sum_{k=K}^{K^{\prime}} \sum_{p} P\left(E_{k, p}\right)<2{ }_{i}^{4}$, since such a circum- 
stance may happen only in the case where one of the corresponding segments is a sub-segment with half length of the other.

Concerning $\Sigma^{2}$, the number of $k$ appearing in the summand is at most Min $\left([\lambda \log k], K^{\prime}\right)$ with some $\lambda>0,{ }^{3)}$ and for each pair $(k, p)$ the number of $p^{\prime}$ appearing there is at most of order $\left(2^{k^{\prime}-k} k\right)^{v}$, which is deduced from Lemma 2. Hence we have

$$
\begin{aligned}
\sum^{2} P\left(E_{k, p} \cap E_{k^{\prime}, p^{\prime}}\right) & \leqq \sum_{k=K}^{K^{\prime}} \sum_{k^{\prime}=k}^{k+[\lambda \log k]}\left(\sum_{p, \nu^{\prime}}^{2} P\left(E_{k, p} \cap E_{k^{\prime}, p^{\prime}}\right)\right) \\
& \leqq O(1) \sum_{k=K}^{K^{\prime}} \sum_{k^{\prime}=k}^{k+[\lambda \log k]}\left(\sum_{p, p^{\prime}}^{2} P\left(E_{k, p}\right) e^{-\alpha k^{\prime}}\right) \\
& =O(1) \sum_{k=K}^{K^{\prime}} \sum_{k^{\prime}=k}^{k+[\lambda \log k]}\left(2^{k^{\prime}-k} k\right)^{N} e^{-\alpha k^{\prime}} 2^{N k} P\left(E_{k, p}\right) \\
& =O(1) \sum_{k=K}^{K^{\prime}} e^{-\alpha k} k^{N} 2^{N k} P\left(E_{k, p}\right) \sum_{j=1}^{[\lambda \log k]}\left(2^{N} e^{-\alpha}\right)^{j},
\end{aligned}
$$

which is less than a certain absolute constant $M$. Combining the obvious inequality

$$
\sum_{K \leqq k<k^{\prime} \leqq K^{\prime}} \sum_{p, w^{\prime}} P\left(E_{k, p}\right) P\left(E_{k^{\prime}, p^{\prime}}\right) \leqq\left\{\sum_{K \leqq k \leqq K^{\prime}} \sum_{p} P\left(F_{k, p}\right)\right\}^{2} \leqq(4 / \delta)^{2},
$$

we have

$$
\sum_{K \leqq k<k^{\prime} \leqq K^{\prime}} \sum_{p, p^{\prime}} P\left(E_{k, p} \cap E_{k^{\prime}, p^{\prime}}\right) \leqq d(4 / \delta)^{2}+M+8 / \delta .
$$

Now let us put $F_{k, p}=E_{k, p} \cap\left(\bigcup_{n=m} E_{n}\right)^{c} . \quad \bigcup_{k=K}^{K^{\prime}} \bigcup_{p} F_{k, p}$ being the subset of $G_{m, l}$, we have

$$
P\left(\bigcup_{k=K}^{K^{\prime}} \bigcup_{p} F_{k, p}\right)<\varepsilon
$$

From (29) and (30), we have

$$
1<\sum_{k=K}^{K^{\prime}} \sum_{p} P\left(F_{k, p}\right) \leqq \sum_{k=K}^{K^{\prime}} \sum_{p} P\left(E_{k, p}\right)<4 / \delta
$$

Applying the Lemma 1 to $F_{k, p}$, we obtain, from (34) and (35),

$$
\underset{K \cong k<k^{\prime} \leqq K^{\prime}}{2} \sum_{p, p^{\prime}} P\left(E_{k, p} \cap E_{k^{\prime}, p^{\prime}}\right) \geqslant 2 \sum_{K \leqq k \leqslant k^{\prime} \leqq K^{\prime}} \sum_{p, p^{\prime}} P\left(F_{k, p} \cap F_{k^{\prime}, p^{\prime}}\right) \geq \frac{1}{\varepsilon}-\frac{4}{\delta} .
$$

Since $\varepsilon$ may be chosen arbitrarily small, (33) and (36) are incompatible. This

\footnotetext{
3) $\lambda$ may be taken as 3 . In fact, we can easily prove that $k^{\prime}<[\lambda \log k]$ implies $r<\left(\lambda_{k} \lambda_{k^{\prime}}\right)^{-1}$,
} 
contradiction proves that $\hat{o}=0$. Hence $P\left(B_{m}\right)=1$ for every $m$, which proves the theorem for the particular case where both $A$ and $B$ are lattice points. This completes the proof.

\section{REFERENCES}

[1] P. Lévy, Processus stochastiques et mouvement brownien, Gauthier-Villars, Paris (1948).

[2] T. Sirao, On the uniform continuity of Wiener process, J. Math. Soc. Japan, vol. 6 (1954), pp. 332-335.

[3] K. L. Chung and P. Erdös, On the application of the Borel Cantelli lemma, Trans. Amer. Math. Soc., vol. 72 (1952), pp. 179-189.

\section{Mathematical Institute}

Aichi-Gakugei University 